\title{
Jejunal inflammatory fibroid polyp: a rare cause of intussusception
}

\author{
Md. Rajibul Haque Talukder, Md. Noor A Alam, Zahid Iqbal Jamal Uddin, Nilufar Shabnam \\ Department of Surgery, Bangladesh Institute of Research and Rehabilitation in Diabetes, Endocrine and \\ Metabolic Disorder (BIRDEM) Dhaka, Bangladesh
}

\begin{abstract}
Inflammatory fibroid polyp is a benign and non-neoplastic condition of the gastro-intestinal tract, commonly affecting the gastric antrum, though it can affect any part of the gastro-intestinal tract. It is a submucosal, sessile, polypoid mass composed of myofbroblast like mesenchymal cells, numerous small blood vessels and marked inflammatory cell infiltrate mainly eosinophils. It commonly presents with intestinal obstruction or intussusception. We present here a case of recurrent episodes of small intestinal sub-acute obstruction due to intermittent intussusception associated with inflammatory ûbroid polyp of jejunum.
\end{abstract}

Ibrahim Med. Coll. J. 2015; 9(2): 58-60

\section{Introduction}

Inflammatory fibroid polyp (IFP) is a relatively rare disorder thought to be clinically and histologically benign and was first described as "polypoid fibroma" in 1920 by Konjetzny and as an eosinophilic granuloma by Vanek in $1949 .{ }^{1}$ It originates from sub mucosa and grows as a polypoid mass. ${ }^{2,3}$ It is an uncommon nonneoplastic proliferating lesion, most commonly occur in the gastric antrum, followed by the small bowel. ${ }^{4}$ However, it can develop in other parts of the gastrointestinal tract. The term "inflammatory fibroid polyp" was first proposed by Ranier and Helwig ${ }^{5}$ in 1953 and is now a generally accepted term.

Clinical symptoms may vary according to the location of the lesion. ${ }^{6}$ If it occurs in the stomach, the most frequently seen symptoms are vomiting, epigastralgia and bleeding. Intussusception and obstruction are symptoms of the lesion located in the small bowel. On the other hand, colicky pain, weight loss, diarrhea, bleeding and anaemia are seen in colonic lesions. Inflammatory fibroid polyps can be found in all age groups but peak incidence is between the sixth and seventh decades. ${ }^{4}$ Although most of the cases are sporadic, a familial relationship has also been described. ${ }^{7}$ We describe here a case of recurrent episodes of small intestinal sub-acute obstruction due to intermittent intussusception associated with inflammatory fibroid polyp of jejunum.

\section{Case Report}

A 60-year-old woman, non-diabetic, normotensive, asthmatic, housewife was admitted to BIRDEM general hospital with the history of frequent colicky abdominal pain initially around the umbilical region and then spread to the whole abdomen for three months and was relieved by anti-spasmodic drugs. She also complained of abdominal distension and vomiting of partially digested food particles, the frequency of which increased up to 4 to 5 times a day. There was also history of occasional constipation for the same duration. On general examination, she was found to be anaemic and dehydrated. Her vital signs were within normal physiological limits. Her abdomen was found to be distended. A firm, mildly tender, elongated mass was palpable in the umbilical region and no other organomegaly was present. Percussion note was tympanic and bowel sound was present. There was no other physical finding. Ultra sonogram revealed an ovoid hypoechoic area in right paraumbilical region; suggested possibilities were gut originated mass or

Address for Correspondence:

Dr. Md. Rajibul Haque Talukder, Registrar, Department of Surgery, Ibrahim Medical College and BIRDEM General Hospital, 122 Kazi Nazrul Islam Aveune, Dhaka-1000, Bangladesh 


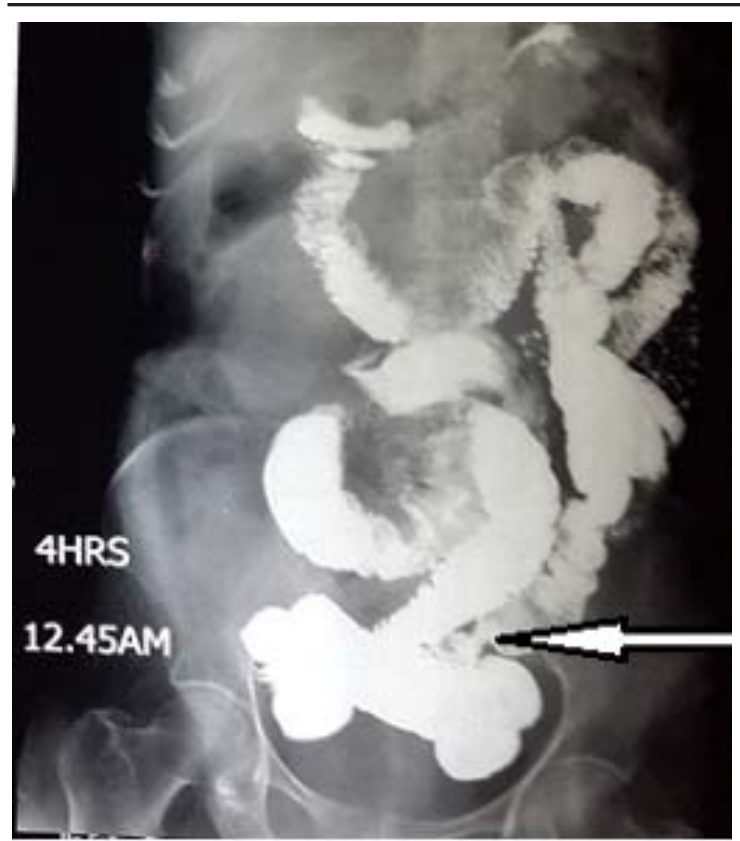

Fig.1: Barium meal follow through showing segmental narrowing in the distal and lower part of jejunum

enlarged lymph node with thick walled distended bowel. Computed tomography (CT) scan revealed thick walled small bowel loops. Barium meal follow through X-ray showed segmental narrowing in the distal and lower part of jejunum in the left side of pelvic cavity (Fig. 1).

Laparotomy revealed that a $20 \mathrm{~cm}$ long portion of the jejunum was congested and particularly firm in consistency. The portion immediately distal to it was

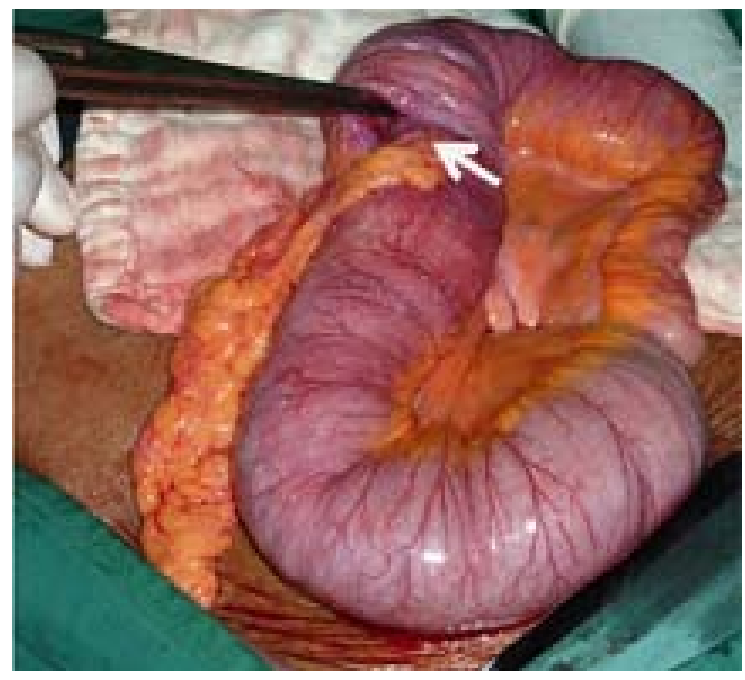

Fig.2: Per operative picture showing the invaginated jejunum

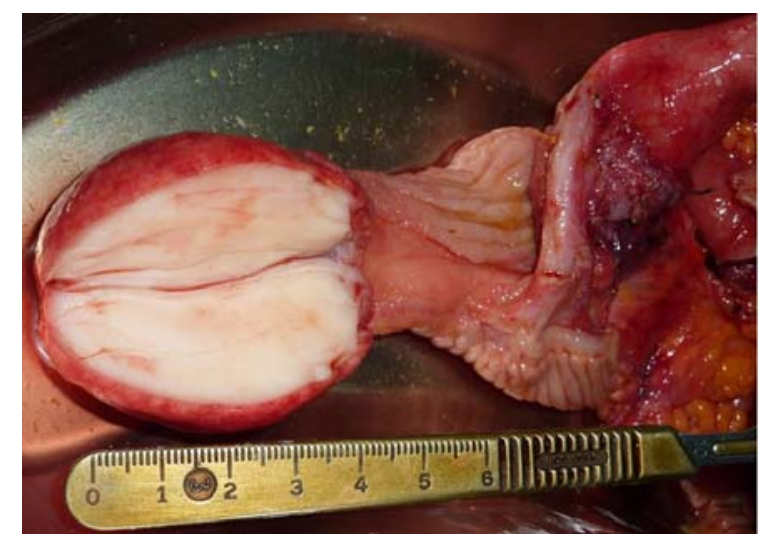

Fig.3: Bisected Specimen of the jejuna polyp

narrowed by a stricture. The bowel had undergone a jejuno-jejunal intussusceptions (Fig 2). Resection of the whole intussuscepted bowel was done and end to end anastomosis was performed. Post operatively the specimen was cut open which confirmed intussusception and a polypoid mass measuring about $4 \times 3 \times 3 \mathrm{~cm}$. The cut surface was white, firm with focal myxoid appearance (Fig 3). Microscopic examination showed sub mucosal tumor with overlying ulcerated jejunal mucosa. The background stroma was sclerotic and contained a fair number of plasma cells, lymphocytes, histiocytes and eosinophils. Based on the operative and histological findings, it was diagnosed as a case of jejunal intussusception due to inflammatory fibroid polyp.

\section{Discussion}

Adult intussusception is caused by a well-definable pathological abnormality in $70-90 \%$ of cases. ${ }^{8}$ In general, benign lesions are the commonest causes of intussusception involving small bowel, accounting for $70 \%$ of cases. ${ }^{8}$ Examples of benign lesions include sub mucosal lipomas, Peutz Jeghers polyps, congenital band adhesions, intussuscepting Meckel diverticulum and inflammatory fibroid polyps. The most common site for inflammatory fibroid polyps is the gastric mucosa, accounting for $70 \%$ of cases. ${ }^{9}$ Of other gastrointestinal sites affected, the small bowel is the most common, accounting for $23 \%$, with the ileum being predominating site. ${ }^{9}$ The colon (4\%), gallbladder, esophagus, duodenum and appendix have also been described as rare sites. ${ }^{9}$ The fifth to seventh decade of life is the most common and both sexes are equally affected. ${ }^{9}$ 
The clinical presentation of an inflammatory fibroid polyp depends on the site of the tumour. Many are asymptomatic and are identified incidentally during endoscopy or laparotomy.$^{10}$ Inflammatory fibroid polyps in the small bowel can present with chronic episodes of colicky abdominal pain, lower gastrointestinal bleeding, anaemia and rarely bowel obstruction due to intestinal intussusception. ${ }^{11}$ In our case there was no history of gastrointestinal bleeding episodes and obstruction. But she had colicky pain, distension of the abdomen and vomiting. The vomiting could be due to the obstruction at the jejunum.

The etiology of inflammatory fibroid polyps is unknown. Theories involving triggers such as foreign body, parasite and chronic $H$. pylori infection have been suggested but remain unsupported. ${ }^{9}$ A poorly controlled inflammatory response to a chemical, traumatic or metabolic mucosal injury has also been hypothesized. ${ }^{10}$ Given its marked eosinophilic infiltration in most cases, a localized variant of eosinophilic gastroenteritis is another proposed aetiology. ${ }^{11}$ We could not ascertain the factors associated with the development of this jejunal inflammatory fibroid polyp in our case. There was no previous report of jejunal intussusception due to inflammatory fibroid polyp from Bangladesh. Our case indicates that this condition should considered in elderly patients with features of chronic abdominal pain, vomiting and frequent distension of the abdomen.

\section{References}

1. Vanek J. Gastric submucosal granuloma with eosinophilic infiltration. Am J Pathol 1949; 25(3): 397-411.
2. Johnstone JM, Moroson BC. Inflammatory fibroid polyp of the gastrointestinal tract. Histopathology 1978; 2: 349-61.

3. Shih L-N, Chang S-L, Chuang S-M, Kuo C-F. Inflammatory fibroid polyp of the jejenum causing intussusception. Am J Gastroenterol 1997; 92: 162-4.

4. De la PR, Picardo AL, Cuberes R, Jara A, MartinezPenalver I, Villanueva MC et al. Inflammatory fibroid polyps of the large intestine. Dig Dis Sci 1999; 44(9): 1810-6.

5. Helwig EB, Ranier A. Inflammatory fibroid polyps of the stomach. Surg Gynecol Obstet 1953; 96: 355-67.

6. Ali J, Qi W, Hanna SS, Huang S-N. Clinical presentations of gastrointestinal inflammatory fibroid polyps. CJS 1992; 35: 194-8.

7. Anthony PP, Morris DS, Vowles KDJ. Multiple and recurrent inflammatory fibroid polyps in three generations of a Devon family: a new syndrome. Gut 1984; 25: 854-62.

8. Yakan S, Caliskan C, Makay O, Denecli AG, Korkut MA. Intussusception in adults: clinical characteristics, diagnosis and operative strategies. Word J Gastroenterol 2009; 15: 1985-9.

9. Wysocki AP, Taylor G, Windsor JA. Inflammatory fibroid polyps of theduodenum: a review of the literature. Dig Surg 2007; 24: 162-8.

10. Rehman S, Gamie Z, Wilson TR, Coup A, Kaur G. Inflammatory fibroid polyp (Vanek'stumour), an unusual large polyp of the jejunum: a casereport. Cases J 2009; 2: 7152.

11. Nonose R, Valenciano JS, da Silva CM, de Souza CA, Martinez CA. Ileal intussusception cause by Vanek'stumour: a case report. Case Rep Gastroenterol 2011; 5: 110-6. 